\title{
Replace Psychometric Inferences with Direct Brain Measurements: LORETA Reflects Traditional Cerebral Loci for Neuropsychological Tests
}

\author{
Paula L. Corradini 1,2,3, Michael A. Persinger ${ }^{1,2,3,4^{*}}$ \\ ${ }^{1}$ Clinical Neuropsychology Laboratory, Laurentian University, Sudbury, Canada \\ ${ }^{2}$ Behavioural Neuroscience Program, Laurentian University, Sudbury, Canada \\ ${ }^{3}$ Department of Psychology, Laurentian University, Sudbury, Canada \\ ${ }^{4}$ Biomolecular Sciences Program, Laurentian University, Sudbury, Canada \\ Email: ${ }^{*}$ mpersinger@laurentian.ca
}

Received 30 July 2015; accepted 5 September 2015; published 8 September 2015

Copyright (C) 2015 by authors and Scientific Research Publishing Inc.

This work is licensed under the Creative Commons Attribution International License (CC BY). http://creativecommons.org/licenses/by/4.0/

\begin{abstract}
Inferences of subtle cerebral injury and dysfunction have been historically dependent upon psychometric tests from which clinical neuropsychological profiles are generated. In addition to being secondary, over-inclusive and crude indicators of cerebral activity, psychometric tests are subject to economic incentives to "re-norm" traditional methods under the pretense of "ensuring" contemporary representations that are sanctioned by regulating organizations dominated by agendas of control over the interpretations of clinicians. The validity of neuropsychological tests is essential for their perspicacious application and interpretations. We measured the quantitative electroencephalographic profiles and calculated s-LORETA (standardized Low Resolution Electromagnetic Tomography) profiles in real time for normal men and women while they engaged in both traditional and novel neuropsychological tests that were employed to infer localized brain injury. Conspicuous alterations in source current density within specific frequency bands occurred within various regions of the right prefrontal region during performance of the Category, Design Fluency and Conditioned Spatial Association Test, the prefrontal medial surface during Toe Graphaesthesia, the caudal medial surface during Toe Gnosis, the left temporal region during Speech-Sounds, and within the right retrosplenial-parahippocampal region for Seashore Rhythms. Results supported the well established regional associations with the classic neuropsychological tests, verified the cerebral localization with more recent procedures, and emphasized the utility of modern real-time, direct cerebral imaging procedures.
\end{abstract}

\footnotetext{
${ }^{*}$ Corresponding author.
} 
Keywords

\section{s-LORETA, Neuropsychological Tests, Category Test, Conditioned Spatial Association Test, Design Fluency Test, Seashore Rhythm Test, Speech Sounds Rhythm Test}

\section{Introduction}

The performance-based procedures ("tests") by which clinical neuropsychologists have inferred the integrity of neuronal function within regions of the human cerebrum have progressed through several stages of constructvalidity. During the late $19^{\text {th }}$ century and early $20^{\text {th }}$ century the associations between behaviours and conspicuous structural alterations within the cerebrum were confounded often by the delays of years to decades between the observation and the post-mortem examination. The development of CT (Computerized Tomography) and MRI (Magnetic Resonance Imaging) permitted more temporally contiguous comparisons between quantitative measurements of specific behaviours and more detailed alterations of signal organization within the cerebral volume. However, these measurements were indicators of structural correlates rather than direct functional or dynamic contributions. Concurrent measurements of the specific class of behaviors and the activity within correlative regions within the cerebrum required the development of the functional tools of imaging such as fMRI, PET (Positron Emission Tomography) and SPECT (Single Photon Emission Computed Tomography). Although remarkably precise in their differentiation of the location of cerebral activity, reflecting perfusion or a highly correlated metabolic product of neuronal and glial activity during specific tasks, the equipment is largely static, cumbersome and expensive [1].

The development of neuroimaging tools has afforded researchers the opportunity to validate neuropsychological tests that are used to infer cerebral functioning. However, few studies have been conducted and even fewer studies employ the use of electroencephalography. Zakzanis et al. [2] attempted to validate the classic trail making test using fMRI. Results from this study indicated that even after modifications were made to the testing procedure there was significant activation in the left frontal regions including the dorsolateral prefrontal cortex and regions involved with motor control. In addition to the trail making tests, common neuropsychological tests that have been studied in this manner include the Stroop test [3] [4], the toe Graphaesthesia test [5], and the California verbal learning test [6].

The convergence of quantitative electroencephalographic technology with algorithms employed to infer activity within the cerebral volume has allowed a more versatile and dynamic paradigm by which the power within the patient's cerebral activity over incremental bands of classical EEG ranges can be imaged within computerized representations of the three axes of the human cerebrum. One of these procedures, s-LORETA (standardized Low Resolution Electromagnetic Tomography), has demonstrated substantial utility within experimental and clinical settings for monitoring the fluidity of changing behaviours within specific contexts [7] [8]. Although the spatial resolution for the simplest of configurations is in the order of $5 \mathrm{~mm}$, the access to measurements of current densities or inferences of "power" across a range of discrete $1 \mathrm{~Hz}$ frequency bands is still revealing and is strongly correlated with the task-specific regions measured by fMRI [9] [10] and PET [11]-[13]. Here we present compelling evidence that s-LORETA profiles of normal volunteers engaged in different classic and novel neuropsychological, performance-based procedures support the construct validity of these tests and reveal a realistic perspective of the volume and variations involved with task execution.

\section{Methods}

\subsection{Subjects}

A total of 10 men and women volunteered for this study according to the guidelines of the university's Research Ethics Board for Human Subject Participation. The Category Test, Seashore Rhythm Test, Speech-Sounds Perception Test, Conditioned Spatial Association Test, and Design Fluency Test were completed by 3 men and 2 women whose mean age was 24.6 years $(\mathrm{SD}=1.8)$. The final two tests, Toe Gnosis and Toe Graphaesthesia tests, were performed by five additional participants ( 3 men and 2 women) whose mean age was 26.0 years ( $\mathrm{SD}=$ 2.5). All ten individuals were attending university and did not display neuropsychological evidence of hypo- 
function or deficits.

\subsection{Neuropsychological Tests}

Seven neuropsychological tests, frequently employed for clinical assessments, were selected. There were three classic tests from the Halstead-Reitan Battery: Category Test, Seashore Rhythm Test, and Speech-Sounds Perception Test, and, four more recent tests: Conditioned Spatial Association, Design Fluency, Toe Gnosis, and Toe Graphaesthesia. According to traditional interpretations the accuracy or speed, compared to normative data, by which a subject completes each test reflects the integrity of function within the right prefrontal (dorsal convexity) region for the Category Test [14] [15] the right superior temporal cortices for the Seashore Rhythm Tests [16], the left superior temporal cortices for the Speech Sounds Test [17] [18], the right orbital frontal region for the Conditioned Spatial Association Test and the right more rostral prefrontal region for the Design Fluency Test [19]. Toe Gnosis was more associated with activity within the medial (paracentral) lobule of the parietal lobe while activity within the medial rostral prefrontal region has been shown by SPECT to be activated during Toe Graphaesthesia stimulation [5].

Our rationale for selecting three tests that traditionally involve the right prefrontal region was to discern the spatial or frequency-dependent differential capacity of s-LORETA within the same gross volume. We reasoned it could be possible for tasks involving the same cerebral region to be dominated by different frequency bands of power as discerned from the QEEG. The two temporal lobe tasks would be considered gross reference measures to reflect the construct validity as well as the face validity of both the "left" (Speech Sounds) and "right" (Seashore Rhythm) bias for these tests.

The Category Test involves the subject selecting from four options of various patterns that reflect a spatial configuration concept [20] while the Design Fluency is a time-dependent task that requires the subject to generate as many different four component combinations of novel patterns using two basic shapes [19]. Conditioned Spatial Association requires the subject to learn which blue light of six identical and proximally clustered blue lights is associated with a specific number from 1 to 6 when one of those lights is activated [21]. The learning is by trial and error. The Speech Sounds Test requires the subject to pair a phoneme with the accurate printed grapheme contained within four different options [22] while the Seashore Rhythm task tests the capacity for the subject to discern if two sequences of tones are the same or different [23].

Historically there has been a relative paucity of tests, except for those by which corpus callosal functions are inferred, concerning the integrity of the medial surface of the cerebral hemispheres. Yet the rostral region of this area is serviced primarily by an arterial system, the Anterior Cerebral Artery, which is particularly prone to the torsional effects from the asymmetrical impact of mechanical energies to the two hemispheres. This is particularly relevant considering that about $20 \%$ of the population displays only a single ACA from which the component to the contralateral hemisphere is derived.

Toe Gnosis, like Finger Gnosis, involves identification of which toe on each foot is touched (dorsal surface) lightly with a stylus without the benefit of visual feedback [24]. The order of the toe stimulation employs the same order that is applied to fingers. Each toe is stimulated five times. Toe Graphaesthesia, like Finger Graphaesthesia, involves printing with a stylus either the number 3, 4, 5, or 6 on the ventral surface of each toe. Each toe is stimulated five times. The normative information has been published for children [24] [25] and adults [26].

\subsection{Data Collection and EEG Recording}

While the participants were engaging in the specific neuropsychological tasks brain activity was measured concurrently by a Mitsar-201 portable QEEG system that was connected to a 19-channel electrode cap (ElectrodeCap International) that contained the 10 - 20 Standard Electrode Placement. Electrode sites included Fp1, Fp2, F7, F3, Fz, F4, F8, T3, C3, Cz, C4, T4, T5, P3, Pz, P4, T6, O1, and O2 that were linked to the ears (A1 and A2) for monopolar recordings. Impedance of all channels was less than $10 \mathrm{kOhm}$. Data were acquired using WinEEG v2.84.44 software with a sampling rate of $250 \mathrm{~Hz}$. A $50 \mathrm{~Hz}$ to $70 \mathrm{~Hz}$ notch filter was used in the WinEEG software for all subjects in order to filter high frequency noise during recording. The EEG record was inspected for movement artifacts; the principal component analyses (PCA) method of artifact correction within WinEEG software was employed where appropriate. 


\subsection{Source Localization (SLORETA)}

Source localization analyses were completed by standardized low-resolution electromagnetic tomography (sLORETA) according to Pascual-Marqui and colleagues [7] [8] on 10 samples of EEG recorded from the 19 channels during periods when the individuals were participating in the neuropsychological tests. The time each test was presented (and engaged) was recorded on the EEG record and employed as an aid for determining where the appropriate segments were extracted. Eyes open and eyes closed baseline data were also collected.

These epochs were divided into $2 \mathrm{~s}$ segments that did not overlap for the purposes of analyses. Cross-spectral analyses were completed on all epochs. Independent t-tests without normalization were utilized within sLORETA to discern source localization throughout the telencephalon for each of the software's defined frequency bands which included delta $(1.5-4 \mathrm{~Hz})$, theta $(4-7.5 \mathrm{~Hz})$, alpha-1 $(7.5$ to $10 \mathrm{~Hz})$, alpha-2 $(10-13 \mathrm{~Hz})$, beta-1 $(13-20 \mathrm{~Hz})$, beta-2 $(20-25 \mathrm{~Hz})$, beta-3 $(25-30 \mathrm{~Hz})$ and gamma $(30$ to $40 \mathrm{~Hz})$.

\section{Results}

When compared to baseline measurements for each individual before the standardized neuropsychological tests were initiated the changes within the software-inferred cerebral space were consistent with the traditional interpretations of test functions as well as the inferences of where any damage or hypofunction might be when standardized tests scores were below normal. The specific patterns of change were extraordinarily similar for all participants. For the sake of brevity and clarity only exemplary profiles for each test are shown. Within the figures yellow indicates marked increase in activation while red indicates less increase in activation for the indicated frequency band. For this procedure blue, which was rarely produced during these tasks, indicates diminished activity. Table 1 shows the primary frequency band in which power was increased during the tasks as well as the Brodmann (BA) area and the centroid for the Montreal Neurological Institute coordinates.

Figure 1 shows the increased activation during the subjects' execution of the Conditioned Spatial Association Test. According to software-indicators, the marked increased gamma frequency power $(t=3.49, p<0.05)$ was localized within the right middle prefrontal gyrus (BA 10). The profiles indicate that the area of marked activation (yellow) occurred within the right prefrontal region, particularly within the orbital area. During the Design Fluency Test (Figure 2) individuals displayed increased activation and power within the alpha-2 band within the right frontal gyrus (BA 11) in comparison to baseline $(\mathrm{t}=4.24, \mathrm{p}<0.01)$.

Figure 3 is an example of the profile while subjects solved the problems in the Category Test. The markedly increased activation in power $(\mathrm{t}=6.99, \mathrm{p}<0.01)$ within the alpha-1 band occurred within BA-10 (11) according to the software. However the sagittal profile indicates a much larger region of the prefrontal cortices was involved with this procedure compared to the Design Fluency and Conditioned Spatial Association tasks. Within the various five subtests of the Category Test, there was a common pattern of secondary increased activation and power within beta range that was most prominent in the left inferior parietal lobe, particularly within the area of the supramarginal gyrus (BA 40). The intricate nature of the functional parietal-prefrontal connections is a well

Table 1. Summary of significant findings for each neuropsychological test, spectral band, cortical regions, and Montreal Neurological Institute (MNI) co-ordinates.

\begin{tabular}{|c|c|c|c|c|c|}
\hline Test & Frequency & Region & $\mathbf{X}$ & $\mathbf{Y}$ & $\mathbf{Z}$ \\
\hline Conditioned Spatial Association & Gamma & BA10 & 35 & 40 & 25 \\
\hline Design Fluency & Alpha-2 & BA 11 & 20 & 60 & 15 \\
\hline Speech Sounds & Theta & BA 39 & -40 & -55 & 25 \\
\hline Category Test & Alpha-1 & BA 10 & 5 & 45 & -25 \\
\hline Seashore Rhythm Test & Beta-1 & BA 30 & 10 & -50 & 0 \\
\hline \multirow{2}{*}{ Right Toe Gnosis } & Alpha-1 & BA 31 & -10 & -25 & 40 \\
\hline & Alpha-1 & BA 7 & -10 & -54 & 45 \\
\hline \multirow{2}{*}{ Right Toe Graphaesthesia } & Delta & BA 9 & -10 & 35 & 22 \\
\hline & Gamma & BA 9 & -10 & 40 & 25 \\
\hline
\end{tabular}




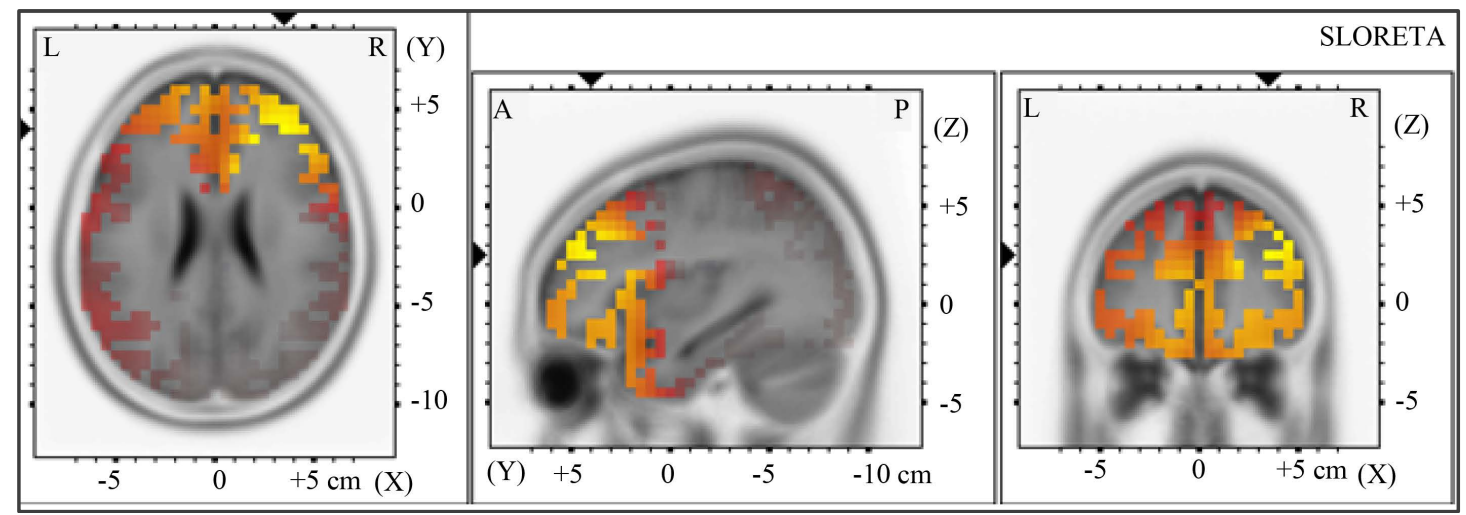

Figure 1. Increased activation compared to baseline recordings during the Conditioned Spatial Association Task in the right middle frontal gyrus (BA 10) in the gamma frequency band.

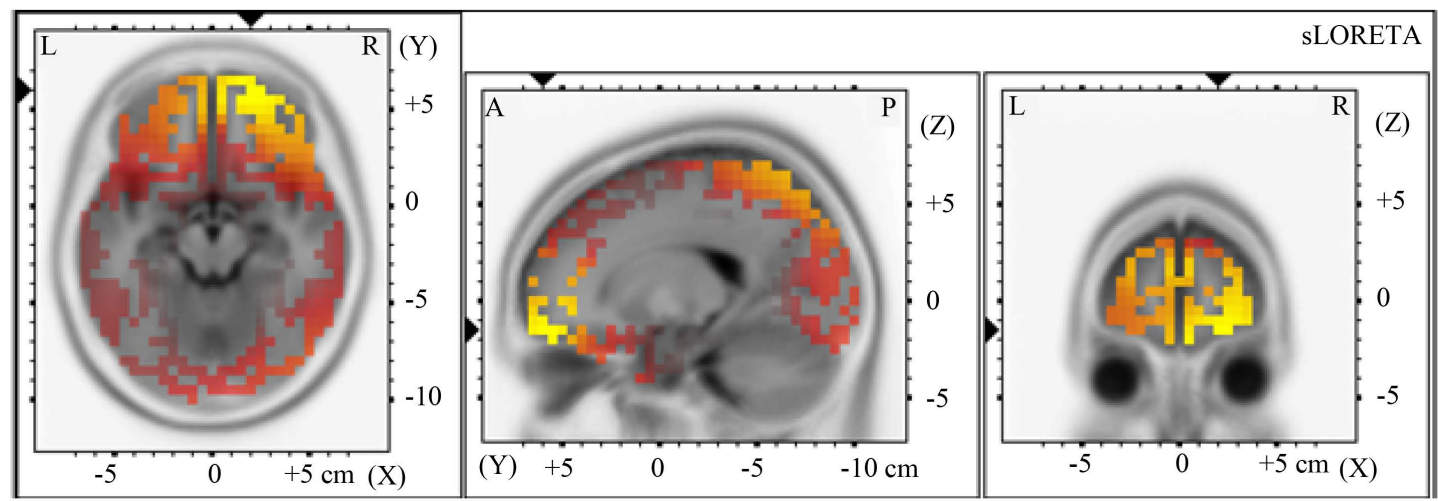

Figure 2. Increased activation compared to baseline recordings during the Design Fluency Test in the right superior frontal gyrus (BA 11) in the alpha-2 frequency band.

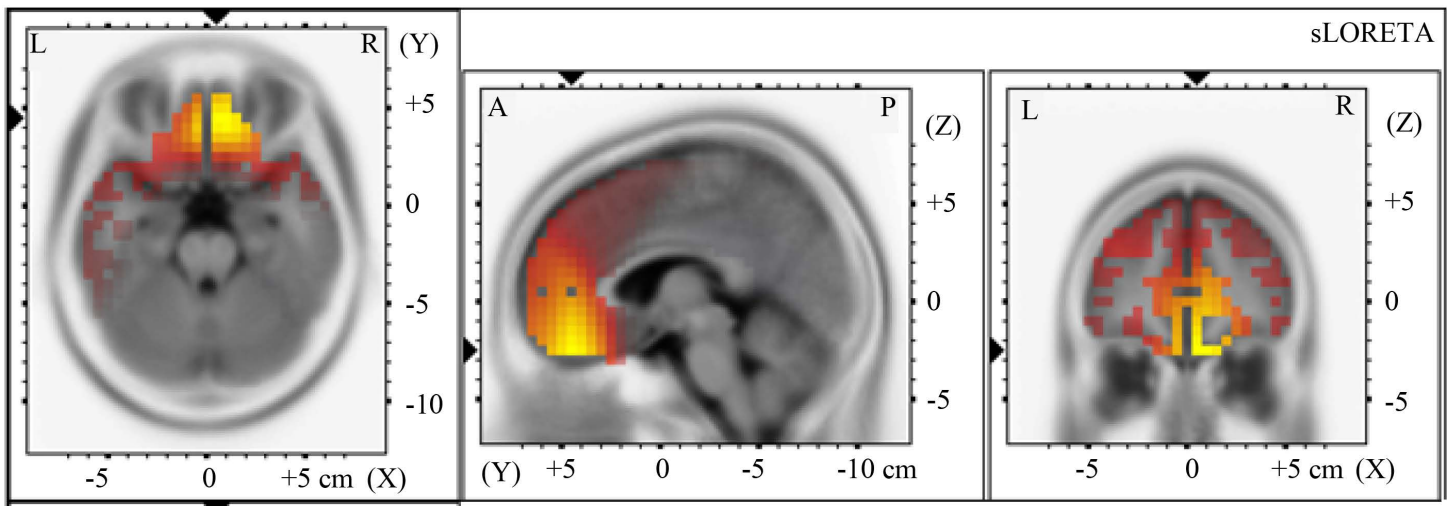

Figure 3. Increased activation compared to baseline recordings during the Category Test in the right orbital gyrus (BA 11) in the alpha-1 frequency band.

known source for the confounding differential for diagnosis of locations in cases of acquired brain injury.

Tasks requiring integration of auditory and cognitive processing were associated with specific changes in activity within regions traditionally associated with these functions. Figure 4 shows the predicted increased activity compared to baseline $(t=4.24, p<0.01)$ within the left temporal lobes during the Speech Sounds Test. Interestingly the increased activity and power was within the theta band within the left superior temporal and adjacent area 39. The generalized activation of the visual cortical area and parietal regions was also noted. A component of this procedure is to visually match the appropriate printed grapheme in a given row of graphemes with 
the phoneme. This low-level generalized activity within the visual cortices was noted for all tests.

Figure 5 shows the profile for the Seashore Rhythm Test. Compared to baseline the significant increase in power $(\mathrm{t}=6.81, \mathrm{p}<0.01)$ within the beta-1 band was primarily within the right hemisphere but not in the temporal cortices which has been typically considered a "weak hypothesis" for inference. Instead source localization indicated it was within the region of the retrosplenial and parahippocampal region. The general area of activation was the smallest of all of the tasks and was associated with the least specificity of tasks according to traditional interpretations.

Figure 6 shows the profile obtained for toe gnosis. As predicted, the greatest change in activity occurred along the caudal medial surface. There were significant differences $(t=9.68, p<0.001)$ found in the left posterior cingulate (BA 31) and left precuneus (parietal lobe: BA 7) region $(t=7.87, p<0.001)$ during the right Toe Gnosis Task for the low-alpha frequency band. The MNI coordinates were: left cingulate: $X=-10, Y=-25$, $Z=40$; left precuneus: $X=-10, Y=-54, Z=45$. For the Toe Graphaesthesia Task (Figure 7) the statistically significant differences were found in the left medial frontal gyrus (BA 9) during the right toe stimulation for both the delta $(\mathrm{t}=4.66, \mathrm{p}<0.001)$ and gamma $(\mathrm{t}=4.41, \mathrm{p}<0.001)$ frequency bands.

\section{Discussion}

The results of direct and real-time measurements of brain activity and source localization for seven different neuropsychology tests that are often employed to discern or infer acquired brain injury support the traditional interpretation of the functions and the integrity of brain regions these tests are assumed to represent [27]. The three tasks that have been inferred to be involved with right prefrontal function, as reflected in patients with CT-verified, MRI-verified or strongly suspected injury in this region, are associated with conspicuous increases relative to baseline of power within frequency bands (alpha to gamma) associated with information processing, organization and concept formation.

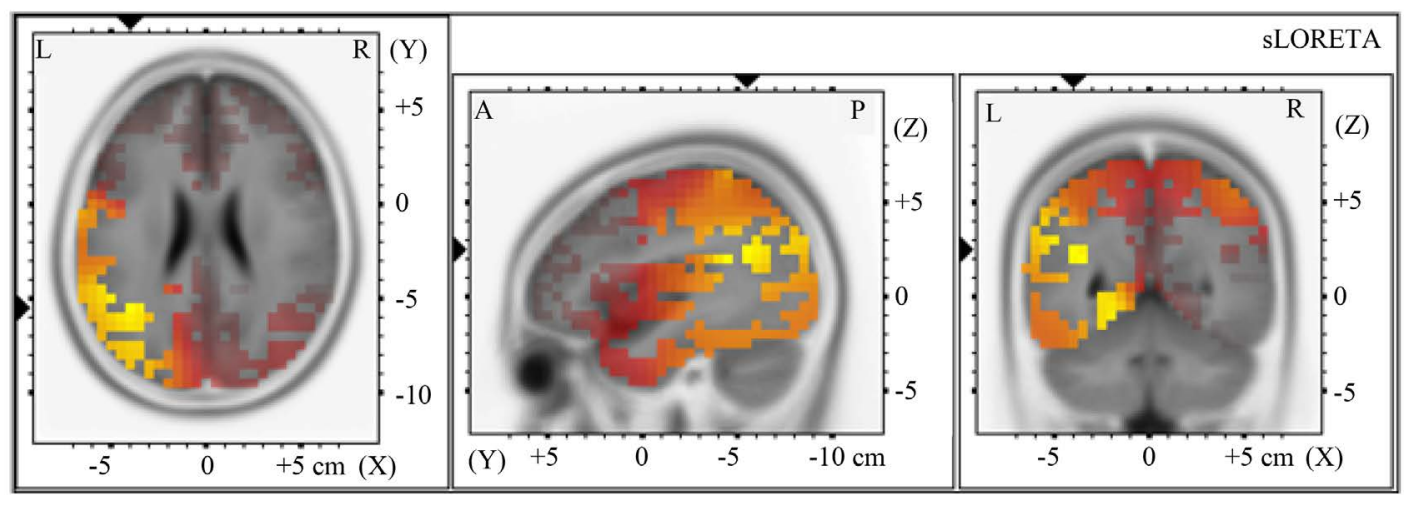

Figure 4. Increased activation compared to baseline recordings during the Speech Sound Test in the left superior temporal gyrus (BA 39) in the theta frequency band.

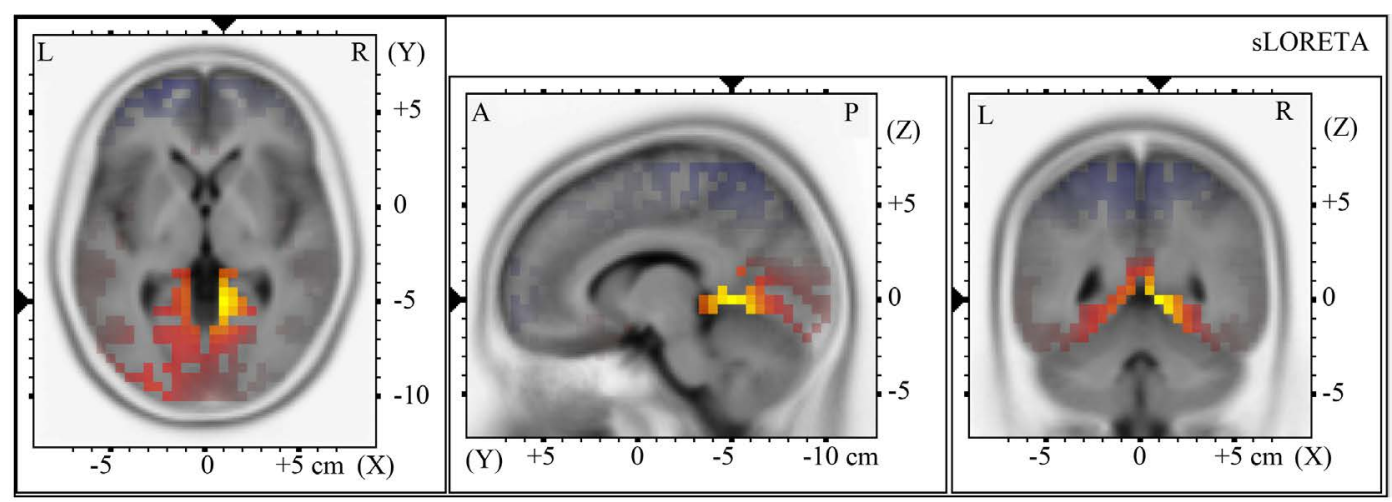

Figure 5. Increased power within the beta 1 range localized primarily within the right Brodman area 30 region (retrosplenial, parahippocampal region) during the Seashore Rhythm Test. 


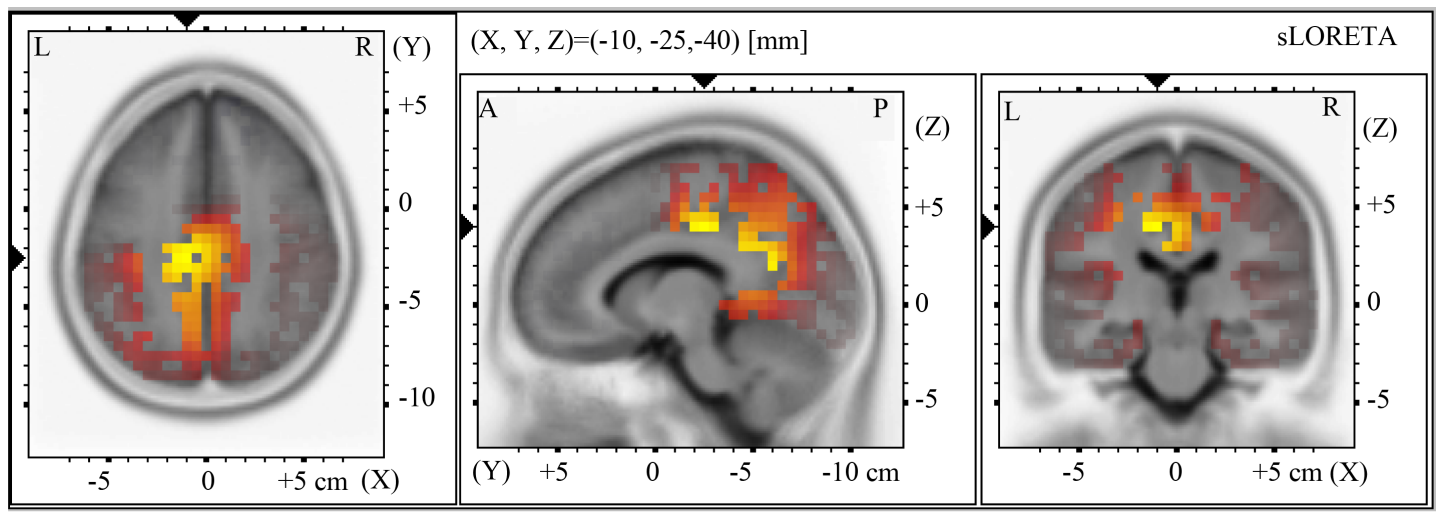

Figure 6. Increased activation compared to baseline recordings during the right Toe Gnosis Test in the left posterior cingulate gyrus (BA 31) adjacent to paracentral medial surface and left precuneus (BA 7) in the alpha-1 frequency band.

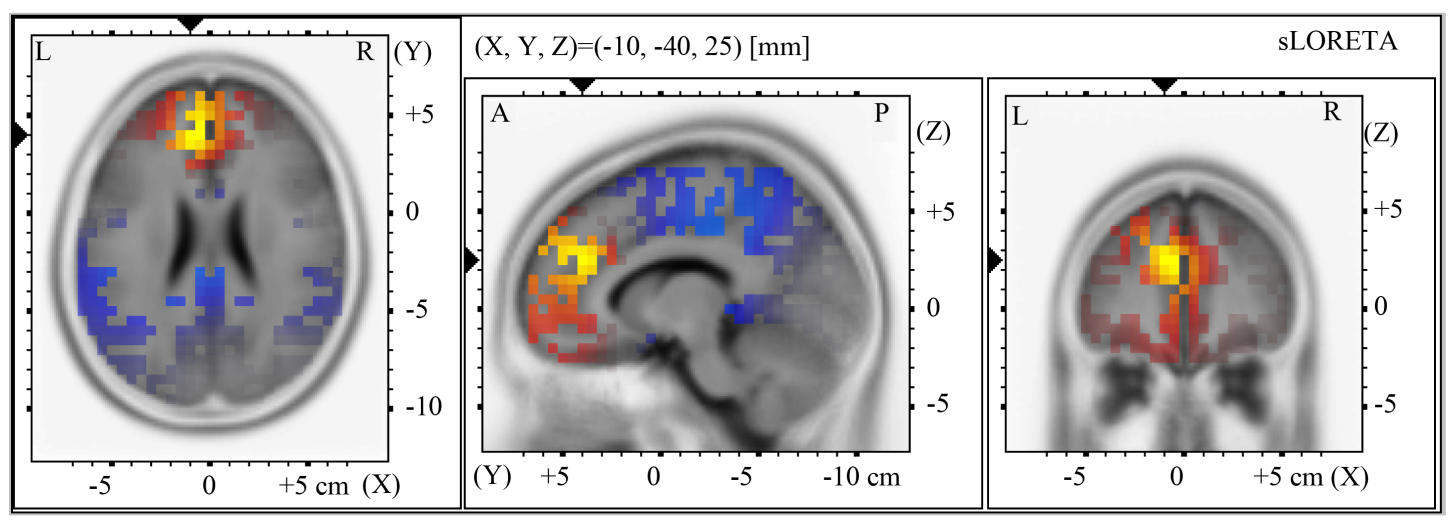

Figure 7. Increased activation compared to baseline recordings during the right Toe Graphaesthesia Test in the left medial frontal gyrus (BA 9) in the gamma frequency band.

The Category Test has often been considered an excellent indicator of diffuse injury within the right prefrontal region [14] [15]. The s-LORETA profiles confirmed the greater involvement of this area during the execution of the task. The area activated most significantly was larger than the areas involved with the other two prefrontal tasks. The Conditioned Spatial Association Task showed discrete activation in the right prefrontal region while the Design Fluency Test, which required recognizing patterns of shapes and placing them over the surface of a paper, also involved the parietal region.

On the other hand, the task (Speech Sounds) that has been attributed to left superior temporal lobe functions [17] [22] is associated with activation in this and immediately adjacent areas. From our experiences, the increased power within theta bands for the Speech Sounds Task is consistent with the over-learned nature associated with this process, even though the manner in which the detailed stimuli (the graphemes and phonemes) are presented may have been partially novel.

The absence of enhanced activity within the right temporal region while normal subjects listen to the Seashore Rhythm Task is consistent with the observation that this procedure, of all of the other tasks, is most vulnerable to distraction [28]. In fact in traditional clinical neuropsychology practice, the attribution of abnormal scores for this task to damage or dysfunction within the right temporal lobe is considered a "weak" hypothesis. Consequently, relatively large lesions in the right temporal lobe, such as those often involved with the population recruited for the validation of this test in the original Halstead series, would be required before the adverse effect influenced the region we measured [29]. Such lesions are likely to involve the parahippocampal region which has ubiquitous connections not only to the temporal lobe but also throughout the cortical manifold.

The simultaneous activation or diminishment of other areas not typically associated with the task might reveal compensatory responses within the cerebrum or even the idiosyncratic organization of functional responses. 
Changes in "power" or source current density within different frequency bands particularly within increments of $1 \mathrm{~Hz}$ could be potentially important for estimating the degree to which the area, activated by specific tasks, was displaying characteristics of novelty or acquired profiles. All of these possibilities would be expected to increase the accuracies of inference and consequently facilitate the application of more appropriate interventions.

We suggest that measuring during active engagement of tasks could reduce the inferential errors that accompanied strictly behavioural or psychometric tasks. When the clinician is dependent completely upon standardized scores and current norms for over-inclusive performances rather than direct measurements of cerebral functions, the superficiality of the tests can obscure the complexity of cerebral dynamics. In many cases the standardized scores are not applicable and have led some clinicians to "not trust the numbers" but rather their "clinical experience" which can be influenced by people who employs them, political agendas by regulating organizations, and theoretical fads. The employment of s-LORETA measurements would require the Clinical Neuroscientist and the Quantitative Medical Practitioner to understand much more about the detailed neuroanatomy, dynamic correlates, electrophysiology, and physics of the human brain in order to increase the precision of diagnostic accuracy and interpretations [30].

\section{Acknowledgements}

We thank Dr. Blake T. Dotta and Professor Kevin S. Saroka for their technical expertise and assistance.

\section{References}

[1] Collura, T.F. (2012) Individualized Assessment and Treatment Using Advanced EEG and Dynamic Localization Techniques with Live sLORETA. International Journal of Psychophysiology, 85, 285-290. http://dx.doi.org/10.1016/i.ijpsycho.2012.07.181

[2] Zakzanis, K.K., Mraz, R. and Graham, S.J. (2005) An fMRI Study of the Trail Making Test. Neuropsychologia, 43, 1878-1886. http://dx.doi.org/10.1016/j.neuropsychologia.2005.03.013

[3] Adleman, N.E., Menon, V., Blasey, C.M., White, C.D., Warsofsky, I.S., Glover, G.H. and Reiss, A.L. (2002) A Developmental fMRI Study of the Stroop Color-Word Task. NeuroImage, 16, 61-75. http://dx.doi.org/10.1006/nimg.2001.1046

[4] Gruber, S.A., Rogowska, J., Holcomb, P., Soraci, S. and Yurgelun-Todd, D. (2002) Stroop Performance in Normal Control Subjects: An fMRI Study. NeuroImage, 16, 349-360. http://dx.doi.org/10.1006/nimg.2002.1089

[5] Persinger, M.A., Webster, D. and Tiller, S.G. (1998) SPECT (HMPAO) Support for Activation of the Medial Prefrontal Cortices during Toe Graphaesthesia. Perceptual and Motor Skills, 87, 59-63. http://dx.doi.org/10.2466/pms.1998.87.1.59

[6] Johnson, S.C., Saykin, A.J., Flashman, L.A., McAllister, T.W. and Sparling, M.B. (2001) Brain Activation on fMRI and Verbal Memory Ability: Functional Anatomical Correlates of CVLT Performance. Journal of International Neuropsychology Society, 7, 55-62. http://dx.doi.org/10.1017/S135561770171106X

[7] Pascual-Marqui, R.D. (2002) Standardized Low-Resolution Brain Electromagnetic Tomography (sLORETA): Technical Details. Methods and Findings in Experimental Clinical Pharmacology, 24, 5-12.

[8] Pascual-Marqui, R.D., Esslen, M., Kochi, K. and Lehmann, D. (2002) Functional Imaging with Low Resolution Brain Electromagnetic tomography (LORETA): A Review. Methods and Findings in Experimental Clinical Pharmacology, 24, 91-95.

[9] Mulert, C., Jäger, L., Schmitt, R., Bussfeld, P., Pogarell, O., Möller, H.J., Juckel, G. and Hegerla, U. (2004) Integration of fMRI and Simultaneous EEG: Towards a Comprehensive Understanding of Localization and Time-Course of Brain Activity in Target Detection. NeuroImage, 22, 83-94. http://dx.doi.org/10.1016/j.neuroimage.2003.10.051

[10] Vitacco, D., Brandeis, D., Pascual-Marqui, R. and Martin, E. (2002). Correspondence of Event-related Potential Tomography and Functional Magnetic Resonance Imaging during Language Processing. Human Brain Mapping, 17, 412. http://dx.doi.org/10.1002/hbm.10038

[11] Dierks, T., Jelic, V., Pascual-Marqui, R.D., Wahlund, L.O., Julin, P., Linden, D.E.J., Maurer, K., Winblad, B. and Nordberg, A. (2000) Spatial Pattern of Cerebral Glucose Metabolism (PET) Correlates with Localization of Intracerebral EEG-Generators in Alzheimer's Disease. Clinical Neurophysiology, 111, 1817-1824. http://dx.doi.org/10.1016/S1388-2457(00)00427-2

[12] Pizzagalli, D.A., Oakes, T.R., Fox, A.S., Chung, M.K., Larson, C.L., Abercrombie, H.C., Schaefer, S.M., Benca, R.M. and Davidson, R.J. (2004) Functional but Not Structural Subgenual Prefrontal Cortex Abnormalities in Melancholia. Molecular Psychiatry, 9, 393-405. http://dx.doi.org/10.1038/sj.mp.4001469 
[13] Zumsteg, D., Wennberg, R.A., Treyer, V., Buck, A. and Wieser, H.G. (2005) $\mathrm{H}_{2}{ }^{15} \mathrm{O}$ or ${ }^{13} \mathrm{NH}_{3}$ PET and Electromagnetic Tomography (LORETA) during Partial Status Epilepticus. Neurology, 22, 1657-1660. http://dx.doi.org/10.1212/01.wnl.0000184516.32369.1a

[14] Fisher, N.J., Deluca, J.W. and Rourke, B.P. (2007) Wisconsin Card Sorting Test and Halstead Category Test Performances of Children and Adolescents Who Exhibit the Syndrome of Nonverbal Learning Disabilities. Child Neuropsychology, 3, 61-70. http://dx.doi.org/10.1080/09297049708401368

[15] Golden, C.J., Moses, J.A., Coffman, J.A., Miller, W.R. and Strider, F.D. (1983) Clinical Neuropsychology: Interface with Neurologic and Psychiatric Disorders. Grune and Stratton, New York.

[16] Lezak, M.D. (1983) Neuropsychological Assessment. 2nd Edition, Oxford University Press, New York.

[17] Bornstein, R.A. and Leason, M. (1984) Item Analysis of Halstead's Speech-Sounds Perception Test: Quantitative and Qualitative Analysis of Errors. Journal of Clinical Neuropsychology, 6, 205-214. http://dx.doi.org/10.1080/01688638408401209

[18] Reitan, R.M. and Wolfson, D. (1989) The Seashore Rhythm Test and Brain Functions. Clinical Neuropsychology, 3, 70-78. http://dx.doi.org/10.1080/13854048908404078

[19] Jones-Gotman, M. and Milner, B. (1977) Design Fluency: The Invention of Nonsense Drawings after Focal Cortical Lesions. Neuropsychologia, 15, 653-674. http://dx.doi.org/10.1016/0028-3932(77)90070-7

[20] Reitan, R.M. and Wolfson, D. (1993) The Halstead-Reitan Neuropsychological Test Battery: Theory and Clinical Interpretation. 2nd Edition, Neuropsychology Press, Tucson.

[21] Milner, B. (1982) Some Cognitive Effects of Frontal-lobe Lesions in Man. Philosophical Transactions of the Royal Society of London Biological Sciences, 289, 211-226. http://dx.doi.org/10.1098/rstb.1982.0083

[22] Reitan, R.M. and Wolfson, D. (1990) The Significance of the Speech-Sounds Perception Test for Cerebral Functions. Archives for Clinical Neuropsychology, 5, 265-272.

[23] Gilandas, A., Touyz, S., Beumont, P.J.V. and Greenberg, H.P. (1984) Handbook of Neuropsychological Assessment. Grune and Stratton, Sydney.

[24] Richards, P.M. and Persinger, M.A. (2004) Agility, Gnosis, and Graphaesthesia for the Toes and Fingers in Children: Normative Data (Ages 7-14). International Journal of Neuroscience, 114, $17-29$. http://dx.doi.org/10.1080/00207450490249365

[25] Knights, R.M. and Norwood, J.A. (1980) Revised Smoothed Normative Data on the Neuropsychological Test Battery for Children. Department of Psychology, Carleton University, Ottawa.

[26] Richards, P.M. and Persinger, M.A. (1992) Toe Graphaesthesia as a Discriminator of Brain Impairment: The Outstanding Feet for Neuropsychology. Perceptual and Motor Skills, 74, 1027-1030. http://dx.doi.org/10.2466/pms.1992.74.3c.1027

[27] Kolb, B. and Wishaw, I.Q. (1990) Fundamentals of Human Neuropsychology. W. H. Freeman and Company, New York.

[28] Hom, J. and Reitan, R.M. (1990) Generalized Cognitive Function After Stroke. Journal of Clinical and Experimental Neuropsychology, 12, 644-654. http://dx.doi.org/10.1080/01688639008401008

[29] Halstead, W.C. (1947) Brain and Intelligence. University of Chicago Press, Chicago.

[30] Persinger, M.A. (1995) Neuropsychologica Brevita: An Application to Traumatic (Acquired) Brain Injury. Psychological Reports, 77, 702-724. http://dx.doi.org/10.2466/pr0.1995.77.3.707 